\title{
A UNIT SYSTEM FOR LABORATORY APPARATUS.
}

By Oscar R. Foster.

In large city high schools, where many pupils study chemistry, it is often a serious problem to supply laboratory apparatus quickly, especially when individual work is done. In order to insure promptness at the beginning of a laboratory period and to minimize the teacher's work in large classes, the writer has devised the system here described. This method of apparatus storage and distribution is not designed to meet the needs of those chemical laboratories where provision is made for placing material in drawers beneath each table.

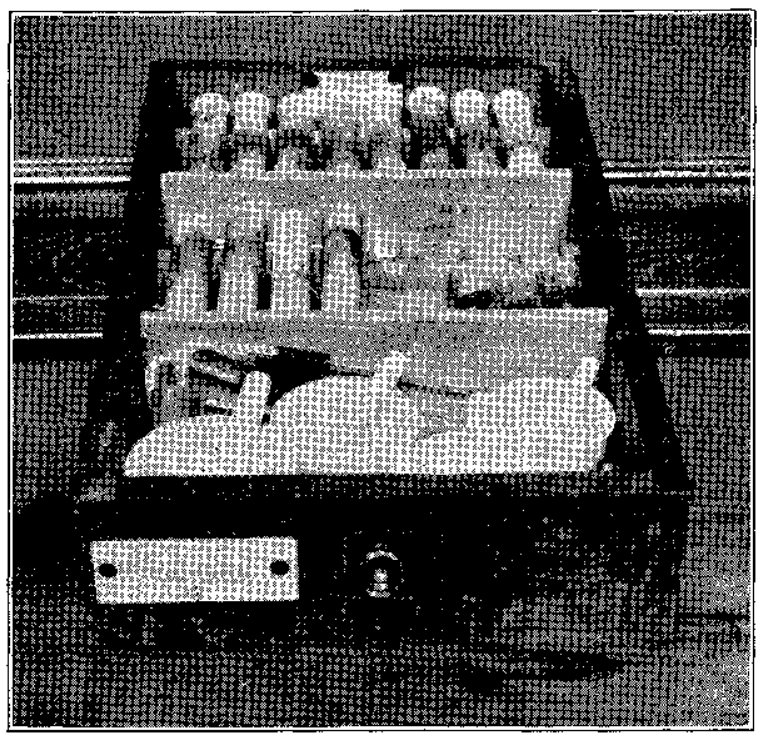

FIGURE 1.

The unit system is as follows: Drawers of convenient size, and as near the laboratory as circumstances permit, are used as containers for apparatus; each drawer constitutes a unit and provides sufficient dismantled apparatus for the performance of one experiment by a class. In some cases, where the quantity of material is larger, two drawers are a unit. The necessary chemicals are kept on the laboratory shelves; only chemicals which are seldom used are stored in the drawers. This system can be extended so as to include the storage of chemicals where conditions make this desirable. Figure 1 and Figure 2 show two small units, Figure 1 for the preparation and properties of 
ammonia, and Figure 2 for the preparation and properties of bromine. The size of these units is about $11 \mathrm{in}$. wide $\times 20 \mathrm{in}$. long $\times 3$ in. deep. For more bulky apparatus, larger units are used. Two sizes are employed, one approximately 11 in. $\times$ 20 in. $\times 6$ in., and another about 11 in. $\times 20$ in. $\times 11$ in. On the inside of the far end of each drawer is placed a sketch of the set-up.

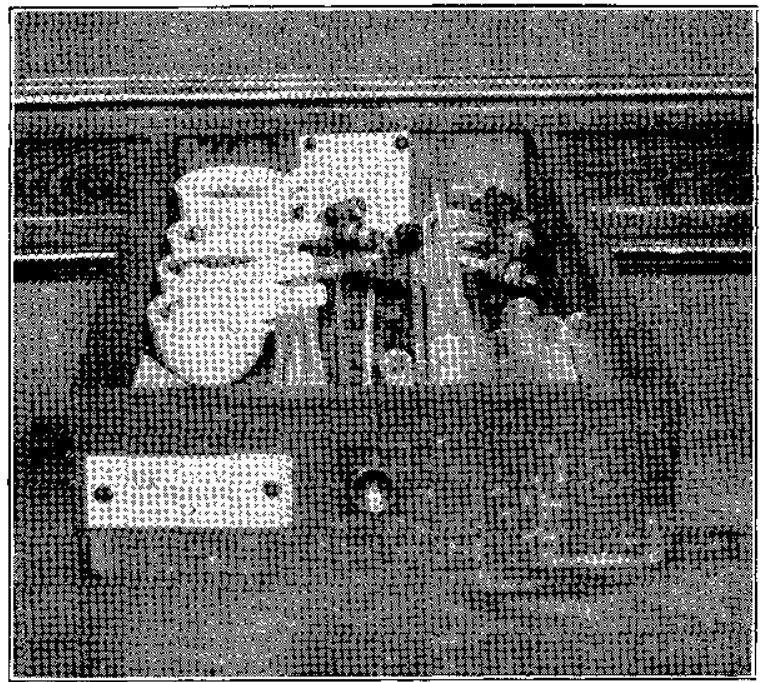

FIGURE 2.

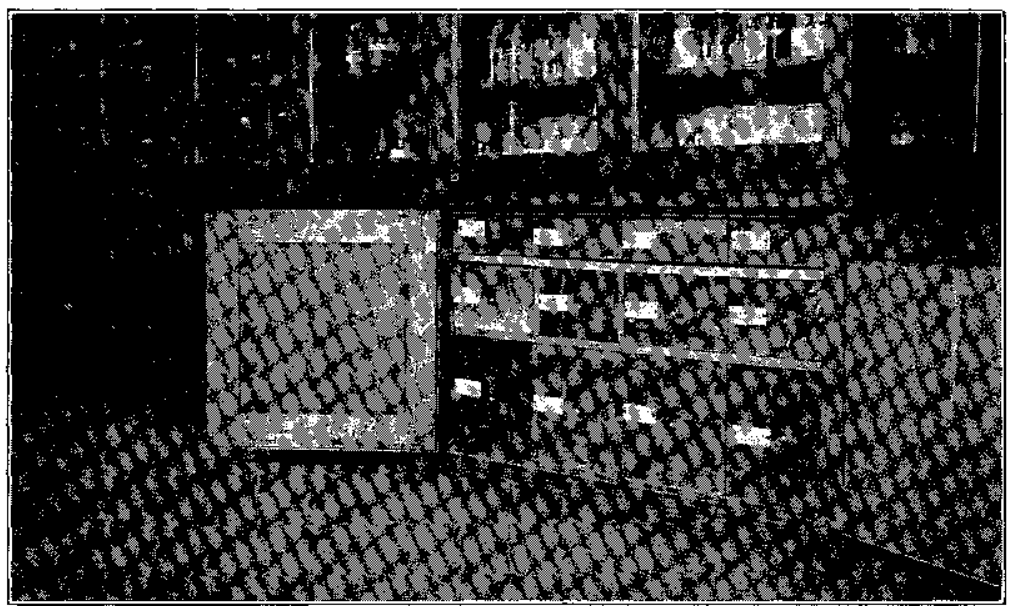

FIGURE 3. 
Figure 3 shows the method of storing the units in hall cases near the chemical laboratory. Each drawer is labeled, its contents being shown in large letters. The entire space required is small. Three compartments as shown in Figure 3 (sufficing for apparatus for a one and a half year course in chemistry) have over all dimensions of $12 \mathrm{ft} .8 \mathrm{in}$. long $\times 27$ in. high $\times 25 \mathrm{in}$. deep.

One feature of the apparatus shown in Figure 1 is worthy of special mention. The test tubes shown in the photograph are fused silica tubes; they are used in this experiment on ammonia to minimize breakage. On account of the low coefficient of expansion, these tubes will not break when there are drops of water on the inside and a Bunsen flame is directed against the outside.

Where large classes are the rule, this system lightens the purely physical work of the teacher. At the beginning of a laboratory period he brings one unit, or at most two units, into the laboratory and places the necessary reagents near them. In high schools where a laboratory assistant is available, the assistant can do this work before or during the change of classes. A brief explanation is given with blackboard illustration; then the student selects sufficient material and performs the experiment. When he has finished he dismantles the apparatus, washes it, and returns it to the drawer. The removal of the apparatus for an entire class is then easily and quickly accomplished.

The method above described possesses the following advantages:

1: Availability - the apparatus is always instantly available.

2. Minimizes effort-only a little work is required to supply material fợ a large class.

3.: Time saving - because the necessary apparatus for an experiment is kept in one place there is no loss of time; such loss will occur if apparatus is widely distributed.

4. Compactness - apparatus for a large number of students can be stored in a small space.

5. Low cost-the cost of containers is small; existing drawers may be used. The apparatus itself can be designed so that it is extremely simple and requires no large or expensive parts.

6. Ease of inspection - the entire stook can be looked over in a short time to detect breakage and make replacements.

The photographs which illustrate this article show part of the equipment in the Manual Training High School of New York City, where this system has been in continuous operation for more than six years. 